\title{
Das Arztzeugnis - Teil 1
}

\section{Iris Herzog-Zwitter ${ }^{a}$, Bruno Soltermann ${ }^{b}$, Andreas Klipstein ${ }^{c}$, Gerhard Ebner}

${ }^{a}$ Dr. iur., Juristin, FMH Rechtsdienst, Bildungsbeauftragte Swiss Insurance Medicine (SIM); ${ }^{b}$ Dr. med., MAS Versicherungsmedizin, Vorsitzender Weiter- und Fortbildungskommission der SIM, Chefarzt Schweizerischer Versicherungsverband; ${ }^{C}$ PD Dr. med. MSc, Leiter Medizin AEH AG, Fachleiter ZAFAS Kurse SIM; ${ }^{d}$ Dr. med. M.H.A., Präsident SIM, Facharzt für Psychiatrie und Psychotherapie, Praxisinhaber, Zürich

\section{Dies ist der Beginn einer kleinen Reihe von Beiträgen, welche die vielfältigen Facet- ten von Arbeitszeugnissen im Spannungsfeld von Medizin und Recht erörtern und Hinweise für den korrekten Umgang geben. Das gerade auch vor dem Hintergrund neuer Aspekte wie etwa Telemedizin.}

\section{Einleitung}

Autoren haben sich zur Beurteilung der Arbeitsfähigkeit einerseits die Frage gestellt «Beurteilung der Arbeitsunfähigkeit - eine Kunst, die niemand kann?» [1], und andererseits lautet der Titel einer weiteren Publikation in der Schweizerischen Ärztezeitung (SÄZ): «Arbeitsunfähigkeitszeugnisse: Ärzte zunehmend im Fokus der Justiz» [2]. Die Unsicherheit war und ist spürbar.

\section{Die Aufgabe des Arztes}

Um den Grad der Arbeitsunfähigkeit beurteilen zu können, ist der Rechtsanwender, sei es die Verwaltung oder das Gericht, auf die Beurteilung der Ärztin angewiesen.

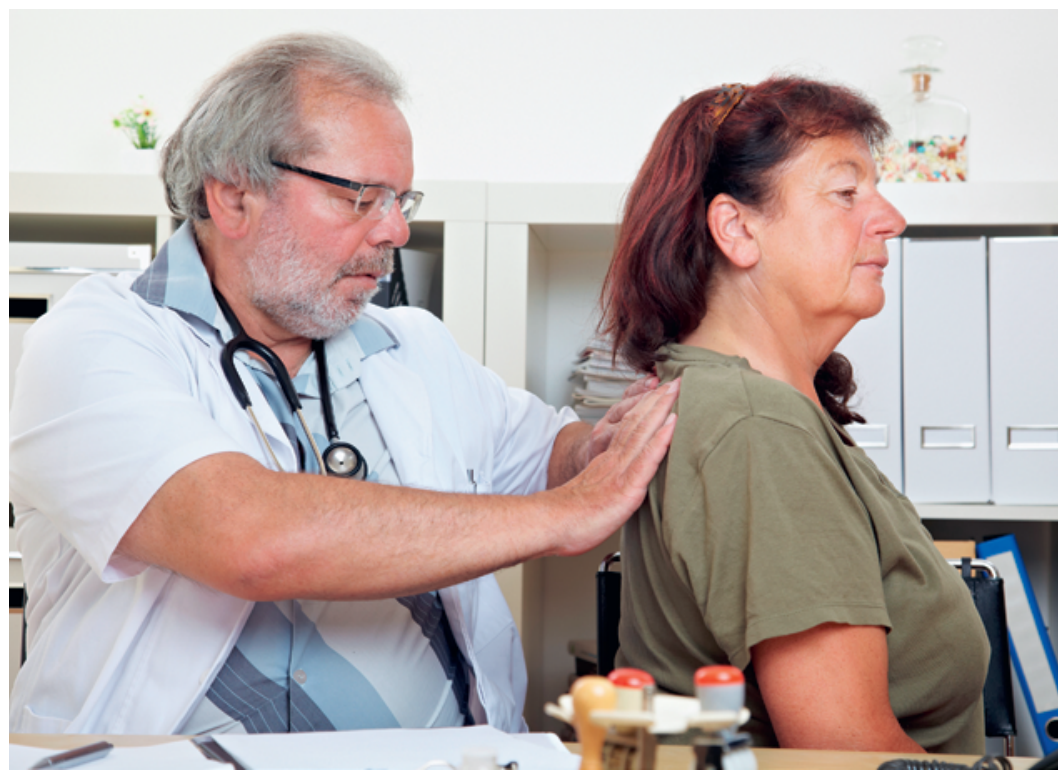

Ärztinnen und Ärzte befinden sich beim Arztzeugnis im Spannungsfeld der Erwartungen von Patientinnen und Patienten, Arbeitgebern und Sozialversicherungen.
«Aufgabe des Arztes oder der Ärztin ist es, den Gesundheitszustand zu beurteilen und dazu Stellung zu nehmen, in welchem Umfang und bezüglich welcher Tätigkeiten die versicherte Person arbeitsunfähig ist. Im Weiteren sind die ärztlichen Auskünfte eine wichtige Grundlage für die Beurteilung der Frage, welche Arbeitsleistungen der versicherten Person noch zugemutet werden können» [3]. Der Arzt hat sich auf medizinische Beurteilungen zu beschränken und keine rechtlichen Schlussfolgerungen zu ziehen [4].

Der Arzt, der einen Arztbericht für einen Auftraggeber zu erstellen hat, muss sich vor Durchführung des Auftrages folgende drei Fragen stellen:

- Wer ist mein Auftraggeber?

- In welchem Rechtsgebiet (Sozialversicherungsrecht oder Privatrecht) führe ich den Auftrag aus?

- Bin ich datenschutzrechtlich ausreichend geschützt und inwieweit bin ich vom Berufsgeheimnis im konkreten Fall entbunden?

Dies sind nur einzelne relevante Fragen, die es zu Beginn des Auftrages zu klären gilt.

Nachfolgend wird dargelegt, dass die Beurteilung der Arbeitsfähigkeit bzw. der Arbeitsunfähigkeit im interdisziplinären Spannungsfeld von Medizin und Recht erfolgt. Verkompliziert wird dieser Vorgang insofern, als der Mediziner einen juristischen Begriff, «die Arbeitsunfähigkeit», medizinisch zu beurteilen hat. Zudem wird die Beurteilung der Arbeitsunfähigkeit mittels Zeugnis unterschiedlich benannt: wie zum Beispiel Arztzeugnis, Arbeitsfähigkeitszeugnis und Arbeitsunfähigkeitszeugnis.

«Das Ausstellen eines Arztzeugnisses ist zwar ein Routinevorgang, aber dennoch eine anspruchsvolle Aufgabe, bei der verschiedene Interessen unter einen Hut gebracht werden müssen. Ärztinnen und Ärzte befinden sich dabei in einem Spannungsfeld zwischen 
ihrer rein ärztlichen Tätigkeit sowie den Erwartungen des Patienten, des Arbeitsgebers und der Sozialversicherungen" [5]. Diese Aussage stammt aus einem SÄZ-Beitrag des Jahres 2014 zum Thema «Das Arztzeugnis im Brennpunkt unterschiedlicher Interessen». Sieben Jahre später hat sich an dieser Aussage nichts geändert.

\section{Arztzeugnis - Verankerung im Gesetz}

Die Problematik beim Arztzeugnis liegt darin, dass es keine Definition des Arztzeugnisses im Gesetz oder in Verordnungen gibt, welche durch die Rechtsprechung anhand von konkreten Fällen einen sogenannten Feinschliff für die Praxis erfahren würde.

Eine Anforderung zum ärztlichen Zeugnis findet sich in Art. 28 Abs. 5 des Arbeitslosengesetzes: «Der Arbeitslose muss seine Arbeitsunfähigkeit beziehungsweise seine Arbeitsfähigkeit mit einem ärztlichen Zeugnis nachweisen.»

\section{Rechtliche Qualifikation des Arztzeugnisses}

In Art. 34 der FMH-Standesordnung wird festgehalten, dass ärztliche Zeugnisse, Berichte und Gutachten als Urkunden gelten. Des Weiteren stellt das Arztzeugnis im strafrechtlichen Sinne eine Urkunde dar, welche bei einer unrichtigen Ausstellung strafrechtliche Konsequenzen nach sich zieht.

\section{Arztzeugnisse und ihr Anwendungsfeld}

Arztzeugnisse haben im arbeitsrechtlichen Kontext eine grosse Bedeutung, sei es im Zusammenhang mit der Lohnfortzahlung des Arbeitgebers oder der Taggeldentschädigung durch Unfallversicherer oder Krankentaggeldversicherer. Arbeitnehmer reichen dem Arbeitgeber ein Arbeitsunfähigkeitszeugnis ein, um ihre Arbeitsunfähigkeit zu beweisen. Die Beweislast liegt gemäss Art. 8 ZGB beim Arbeitnehmer. Ein wichtiger Faktor spielt dabei das Vertrauensverhältnis zwischen Arbeitgeber und Arbeitnehmer. Dieses wirkt sich auch darauf aus, wann der Arbeitgeber das Einreichen eines Arbeitsunfähigkeitszeugnisses verlangt. Oftmals verlangen Arbeitgeber nach einem oder drei Tagen ein ärztliches Attest. Aktuell wurde der Bundesrat im Rahmen eines Postulats (13.3224 von Ruth Humbel) beauftragt, alternative Finanzierungsquellen zur Ausstellung eines Arztzeugnisses zu prüfen und diese aufzuzeigen. In diesem Kontext sieht der Bundesrat die telefonische Ausstellung des Arztzeugnisses als Ansatzmöglichkeit für Sparmöglichkeiten. Telefonisch ausgestellte
Arztzeugnisse sollen vermehrt zum Zug kommen, insbesondere bei leichteren Fällen. In diesem Zusammenhang ist darauf aufmerksam zu machen, dass digital ausgestellte Arztzeugnisse zunehmend ihren Platz in der Versicherungsmedizin einnehmen, unterstützt durch die fortschreitende Digitalisierung, die die Covid-19-Pandemie mit sich bringt [6].

\section{Anforderungen an das Arztzeugnis}

Das Arztzeugnis bestätigt eine Arbeitsunfähigkeit bzw. eine Arbeitsfähigkeit. Je höher der Grad der Arbeitsunfähigkeit ist und je länger diese andauert, umso grösser ist das Risiko einer Berentung.

An die Form werden vom Gesetz keine Anforderungen gestellt, aber aus beweisrechtlichen Gründen ist es angezeigt, ein Arztzeugnis schriftlich auszustellen [7] Um beweiskräftig zu sein, soll ein Arztzeugnis Mindestangaben wie das Ausstellungsdatum, Beginn der Behandlung bzw. des Konsultationstermins, konkrete nachvollziehbare, unmissverständliche Aussagen zur Dauer und zum Umfang der Arbeitsunfähigkeit enthalten und den Empfänger nennen. Nicht erwähnt werden darf in einem für den Arbeitgeber bestimmten Arztzeugnis die Diagnose, es sei denn die Ärztin wurde von der Patientin oder dem Patienten ausdrücklich von der

\section{Das Arztzeugnis stellt im strafrechtlichen} Sinne eine Urkunde dar.

ärztlichen Schweigepflicht entbunden. «Nicht beweisbildend sind in der Regel Arztzeugnisse, die sich allein auf die Patientenschilderungen abstützen und ohne eigene objektive Feststellungen des Arztes bzw. der Ärztin oder erst Monate später ausgestellt werden» [8]. Die neue bundesgerichtliche Rechtsprechung im Sozialversicherungsrecht fordert bei Vorliegen psychischer Erkrankungen für die Beurteilung der Arbeitsfähigkeit einer versicherten Person die Prüfung der Standardindikatoren, die «- unter Berücksichtigung leistungshindernder äusserer Belastungsfaktoren einerseits und Kompensationspotentialen (Ressourcen) anderseits - erlauben, das tatsächlich erreichbare Leistungsvermögen einer versicherten Person einzuschätzen" [9]. Eine solche umfassende Beurteilung, wie sie die Standardindikatoren gemäss BGE 141 V 281 vorsehen, setzt ein vertieftes versicherungspsychiatrisches Wissen voraus und kann im vom Bundesgericht geforderten Ausmass von einem ausgebildeten Gutachter, jedoch kaum vom Grundversorger erwartet werden. So hat der Arzt, die Ärztin gemäss BGE 141 V 281 unter anderem Stellung zu Behandlungs- und Eingliederungserfolg oder -resistenz und zur Persönlichkeit zu nehmen; ferner wird eine Beurteilung der Konsis- 
tenz erwartet. Der Grundversorger ist aber nicht von der Pflicht entbunden, die Standardindikatoren zu kennen, und dort, wo er über die fachlichen Grundlagen verfügt, diese in seiner Beurteilung der Arbeitsfähigkeit zu berücksichtigen.

\section{Zeitpunkt der Ausstellung des Arztzeugnisses}

Arztzeugnisse sollten nicht rückwirkend ausgestellt werden. Nur im Ausnahmefall und im Einzelfall kann ein Arztzeugnis rückwirkend ausgestellt werden höchstens ein paar Tage, allerdings muss die Nachvollziehbarkeit und Verifizierbarkeit gegeben sein. Aber auch dann ist ein Arzt gut beraten, das Zeugnis nur in zuletzt zum eigenen Schutz; andernfalls könnte ein Arztzeugnis, welches rückwirkend ausgestellt wurde, als Gefälligkeitszeugnis angesehen werden, und dies könnte strafrechtliche oder standesrechtliche Konsequenzen nach sich ziehen [10].

\section{Begriff der Arbeitsunfähigkeit im Sozialversicherungsrecht - Verankerung im Gesetz}

Die Arbeitsunfähigkeit ist in Art. 6 des Allgemeinen Teils des Sozialversicherungsgesetzes (ATSG) umschrieben. Der Begriff der Arbeitsunfähigkeit ist ein juristischer Begriff, aber die medizinische Beurteilung nimmt der Mediziner vor. «Es ist eine tatsächliche Frage, die insbesondere von der Ärztin oder vom Arzt zu beantworten ist» [11].

Unter Arbeitsunfähigkeit (AUF) versteht man die durch eine Beeinträchtigung der körperlichen, geistigen oder psychischen Gesundheit bedingte, volle oder teilweise Unfähigkeit, im bisherigen Beruf oder Aufgabenbereich zumutbar Arbeit zu leisten. Bei langer Dauer wird auch die zumutbare Tätigkeit in einem anderen Beruf oder Aufgabenbereich berücksichtigt.

Sowohl der bisherige Beruf als auch der bisherige Aufgabenbereich ist damit angesprochen. Unter die Tätigkeit im Aufgabenbereich wird insbesondere die Tätigkeit im Haushalt und die der Erziehung der Kinder verstanden. Gemäss Art. 6 ATSG ist für die Bemessung der Arbeitsunfähigkeit der Begriff der Zumutbarkeit ein wichtiges Kriterium.

Für den Erhalt des Arbeitsplatzes oder die Reintegration in eine Arbeitsstelle ist es ratsam, von der Arbeitsfähigkeit zu sprechen, da der Arbeitgeber wissen muss, welche Arbeit der Arbeitnehmende leisten kann. Hierfür gibt es das Ressourcenorientierte Eingliederungsprofil (REP) [12] und für die Beurteilung Ausnahmefällen und sehr restriktiv auszustellen, nicht

der Arbeitsfähigkeit das Arbeitsfähigkeitszeugnis der Swiss Insurance Medicine (Arztzeugnis bei Krankheit und Unfall) [13].

\section{Begriff der Arbeitsunfähigkeit im Privatversicherungsrecht}

Der Begriff der Arbeitsunfähigkeit im Privatversicherungsrecht ist in den zugrundeliegenden Allgemeinen Versicherungsbedingungen definiert. Auch wenn der Mediziner die Arbeitsunfähigkeit aus rein medizinischer Sicht zu beurteilen hat, ist es empfehlenswert, die Definition der Arbeitsunfähigkeit in den Allgemeinen Versicherungsbedingungen durchzulesen bzw. diese beim Auftraggeber einzuholen. Im Privatversicherungsrecht spielt insbesondere die Beurteilung der Arbeitsfähigkeit im Haftpflichtrecht bei Personenschäden und im Taggeldbereich eine wichtige Rolle.

\footnotetext{
Literatur

1 Ott R. Beurteilung der Arbeitsunfähigkeit - eine Kunst, die niemand kann? Schweiz Ärzteztg. 2019;100(3):44.

2 Rudolph R. Arbeitsunfähigkeitszeugnisse: Ärzte zunehmend im Fokus der Justiz (Teil 1). Schweiz Ärzteztg. 2010;91(22):864.

3 Urteil Bundesverwaltungsgericht vom 27.3.2020 (C-7009/2018) E. 5.4.4.

4 Rechtliche Grundlagen im medizinischen Alltag FMH/SAMW. Ein Leitfaden für die Praxis. S. 142.

5 Eva Ebnöther. Das Arztzeugnis im Brennpunkt unterschiedlicher Interessen. SÄZ-Podiumsdiskussion. Schweiz Ärzteztg. 2014;95(4):109.

6 www.bag.admin.ch/bag/de/home/das-bag/aktuell/medienmitteilungen.msg-id-80805.html (letzter Zugriff am 27.12.2020).

7 www.fmh.ch/files/pdf23/standesordnung-september-2019 de.pdf (letzter Zugriff am 20.1.2021).

8 Entscheid des Verwaltungsgerichts des Kantons Zürich vom 30.9.2015 (VB.2014.00739) E. 5.2.

9 BGE 141 V 281 E. 2; E. 3.4-3.6 und 4.1.

10 Siehe Art. 34 FMH Standesordnung, wonach die Ausstellung von Gefälligkeitszeugnissen unzulässig ist.

11 Kieser, ATSG Art. 3 Rz. 17.

12 rep.compasso.ch/ (letzter Zugriff am 20.1.2021).

13 www.swiss-insurance-medicine.ch/de/fachwissen-und-tools/ arbeitsunfahigkeit/sim-arbeitsfahigkeitszeugnis (letzter Zugriff am 20.1.2021).
}

\section{Bildnachweis}

Robert Kneschke | Dreamstime.com (Symbolbild)

\section{Das Wichtigste in Kürze}

- Das Arztzeugnis stellt im strafrechtlichen Sinne eine Urkunde dar, welche bei einer unrichtigen Ausstellung strafrechtliche Konsequenzen nach sich zieht.

- Arztzeugnisse sollten grundsätzlich nicht rückwirkend ausgestellt werden.

- Massgebende Kriterien für den Beweiswert eines Arztzeugnisses, sind die Nachvollziehbarkeit und die Verifizierbarkeit.
Dr.iur. Iris Herzog-Zwitte EMH Abteilung Rechtsdienst Nussbaumstrasse 29 Postfach 300 CH-3000 Bern 15 iris.herzog[at]fmh.ch
- Für den Erhalt des Arbeitsplatzes oder die Reintegration in eine Arbeitsstelle ist es ratsam, von der Arbeitsfähigkeit zu sprechen. Der Arbeitgeber weiss dann, in welchem Ausmass dem Arbeitnehmer die Arbeit zumutbar ist bzw. welche Arbeit der Arbeitnehmer leisten kann. 